\title{
Die Streifentechnik für direkt am Körper anmodellierbare Stahl-Leder-Apparate.
}

\author{
'lechnisclıe Mitteilung.
}

Von

Dr. Julius Fuchs - Barden-Baden.

Mit 4 Abbildungen im Text.

(E'ingegangen um 7. De:arbber $19: 1$. )

Die bisherigen Apparate nach dem Hessingschen System und dessen Modifikationen besitzen verstellbare stahlschienen und Hülsen alus Walkleder. Die Improvisationen der Hessingsehen Apparate, wie sie zu orthopärlischen Zwecken notwendig sind, zeigin meist nur eine geringe Haltbarkeit und mangelhafte Elastizitït der Hülsen.

Apparate nach Hessingscher Bauart machen stets einen umständlichen Walkprozeß des Lerkers und eine Bearbeitung der ILetallschirnen üher Cripsmodellen notwendig. Die Herstellung nimmt daher längere Zeit in Anspruch. Sie erfordert bestimntes Material (vernichelten Stahl, Walkleder, Gurten, Garnicrung). Darlureh werden auch die Kosten für solche Apparate verhiltnismäßig hor'h, ganz ahgessehen davon. daß es nicht gelingt, beispiclsweive in Fillen von Knorhenbrüchen oder Gelenkerkrankungen rech tzeitig ein cinwandfreies orthopäuisch-technisches Hilfsmittel zu schaffen. Bei einem Defelitwerlen der Schienen oder Hülsen wird der gewöhnliche Hessingapparat moist ganz unbrauchbar orler erfordert sehr umstädliche Reparaturen. Infolgerlessen sind die Träger der Hessingapparate bis zur Fertigstellung der Reparatur mcist hilflos oder mindestens auf den Gebrauch eines Reserveapparates angewiesen.

Die Hessingapparate lassen sich auBerdem nur schwer umändern und müssen in der Regel bei Anderung des körperlichen Zustandes neu angefertigt werden.

Diesen Nachteilen beugt die Streifentechnik vor. Sie besteht darin, daB das Schienensystem infolge seiner Zusammensetzbarkeit aus elastischen Streifen jeder beliebigen Form und Belastung unmittelbar am Körper angepaßt und mit fabrikmäßig herstellbaren Gelenken fest verbunden werden kann. Die Zusammensetzbarkeit der Schienen aus Längsstreifen ermöglicht eine verhältnis- 
mäßig leichte und stabile, dabei elastische, nach Notwendigkeit auch starre Bauart.

Das Hülsensystem zeichnet sich ron den bisher verwendeten Hülsen und deren Abarten (Spangen) darlureh aus, daß es eines Walkprozesses zur Modellierung nicht berlarf und gleichmäßig elastisch ist. Als Material kann z. B. weiches Kalbleder verwendet werlen. Die einzelne Hülse wird in eine bestimnte Anzahl Querstreifen zerlegt. Die Querstreifen sind jeweils in einem oder beiclen Iängssehienensystemen durch Finklemmen und durch Befestigung mit Stecknieten

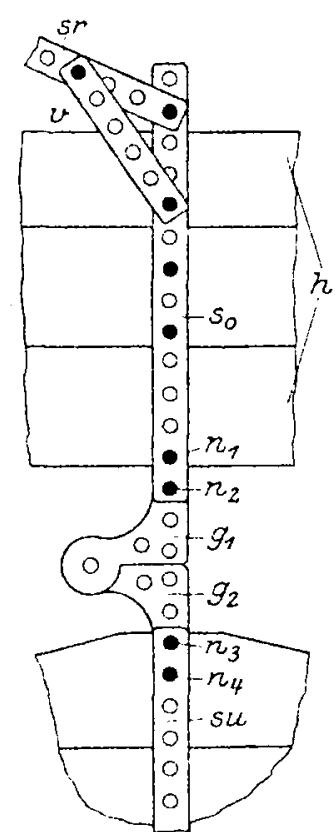

Abb. 1.

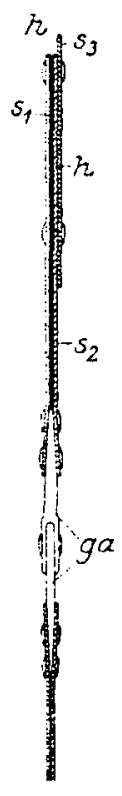

Abh, 2. anzubringen. Durch Schnürung werkon die Querriemen ringartig geschlossen.

Die Verwendungsmöglichkeit der Apparatteile ist infolge der unbegrenzten Zusammensetzbarkeit sehr vielseitig: Es lassen sich außer dem eigentlichen Schienen - Hülsenapuarat nach Hessing auch Korsetts, PlattfuBapparate, künstliche Glicdmaßen oder Teile derselben dem jeweiligen Körperzustand entsprechend ohnt: Zuhilfenahme von Moclellen den Apparatträgern unmittelbar anpassen ${ }^{1}$ ).

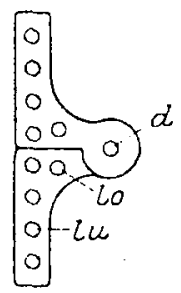

Abh. 3.

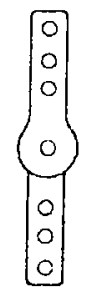

Abb. 4.

Auf den Znichnungen ist ein Streifenapparat beispielsweise in einer tusführung dargestellt, umbl zwar zeigen Abb. 1 und 2 die Anordnung und Befretigung:art der Schienen- und Hülsenteile mit- und untereinander.

In Abb. 1 sind die Śchienensysteme (so und su) mit einem Stahlgelenk (ge 1 und $g$ 2) rlutch Niete ( 1 und $\mathrm{n} 2$ ) und $(\mathrm{n} 3$ und $\mathrm{n} 4$ ) verbunden. Der durch die Verstrebung (v) mit rer Längsschiene (s $(0)$ unbeweglich rerbundene sitzring $(s \mathrm{r}$ ) dient als Beispiel einer weiteren Kombinationsmöglichkeit der Schienen und zugleich als Beispiel einer Querverstrebung der Stützschienen. Der obere und untere Teil des Gelenkes (ga) ist, wie aus Abb. 2 ersichtlich, konizch gestaltet. Das Schienensystem ( $\left.s_{0}\right)$ besteht, wie Abb. 2 zeigt, aus den Metallstreifen (s 1 und s 2) und ist im oberen Teil durch den Metallstreifen (s 3) beispielsweise

1) Die normalisierten Apparatteile werden in Interesse der Billigkeit und (G]eich. mäßigkeit des Materials demnächst fabriknüßig hergestellt. Nach Erledigung des Patentverfahrens wird die Herstellung de! ,. Streifenapparate" (demonstriert am 28. 11. 1921 in der Berlinel (nthopüdischen (Gesellschaft) den Facthorthopëcten auf Antrag frei überlassen. 
414 Fuchs: Die Streifentechnik f. direkt am Kö̈per anmodellierbare Stahl-Leder-Apparate.

verstärkt. Das Hülsensystem (h) ist zwischen elen Schienen (s 1 und s 2) cingeklemmt und eingenietet.

$A b b .3$ zeigt das in Abb. 1 rückgelagerte cingefräste Scheibengelenk (g $1 \mathrm{uml} g \mathrm{~g}$ ) in der vorgelagerten Stellung. Um eine Verlagerung des Irehpunktes (d) z.11 crmöglichen, wird eim- und dasselbe Metallgelenk einfach um rie renkrechte Achse gedreht. Fine höhere oder tiefore Stellung des Drehpunktes kann durch Vernieten mit den Śchienen an den Lörhern (lo und l u) crreicht wrorden.

Abb. 4 zeigt das Gabelgelenk ( $g$ a) aus Abb. 2 in Seitenansirht.

Elastische Stahlschicnen hat bereits Steinmann zur Schienennagelextension bei Frakturen verwendet. Evler-Treptow wandte Chromleder mit. Mr.tallrohrgestïngen an.

I) Strcifentechnik trïgt auch der Körperform Rechnung und ernüglicht. cs, ganz unauffillige. außerordentlich leichte, rasch herstellhare und billige orthopädische Apparate anzufertigen, die den Hessingschen in vielen Fillen sograr überlegen sind. Ausführliche Beschreibung der speziellen rechnik folgt. 